29. Marty P, Roquilly A, Vallee F et al (2013) Lactate clearance for death prediction in severe sepsis or septic shock patients during the first 24 hours in Intensive Care Unit: an observational study. Ann Intensive Care 3:3

30. Mizock BA, Falk JL (1992) Lactic acidosis in critical illness. Crit Care Med 20:80-93

31. Nguyen HB, Loomba M, Yang JJ et al (2010) Early lactate clearance is associated with biomarkers of inflammation, coagulation, apoptosis, organ dysfunction and mortality in severe sepsis and septic shock. J Inflamm (Lond) 7:6

32. Nguyen HB, Rivers EP, Knoblich BP et al (2004) Early lactate clearance is associated with improved outcome in severe sepsis and septic shock. Crit Care Med 32:1637-1642

33. Revelly JP, Tappy L, Martinez A et al (2005) Lactate and glucose metabolism in severe sepsis and cardiogenic shock. Crit Care Med 33:2235-2240

34. Ritz JP, Buhr HJ (2011) Acute mesenteric ischemia. Chirurg 82:863-866, 868-870

35. Ritz JP, Germer CT, Buhr HJ (2005) Prognostic factors for mesenteric infarction: multivariate analysis of 187 patients with regard to patient age. Ann Vasc Surg 19:328-334

36. Rivers E, Nguyen B, Havstad S et al (2001) Early goal-directed therapy in the treatment of severe sepsis and septic shock. N Engl J Med 345:1368-1377

37. Sauerland S, Agresta F, Bergamaschi R et al (2006) Laparoscopy for abdominal emergencies: evidence-based guidelines of the European Association for Endoscopic Surgery. Surg Endosc 20:14-29

38. Severin PN, Uhing MR, Beno DW et al (2002) Endotoxin-induced hyperlactatemia results from decreased lactate clearance in hemodynamically stable rats. Crit Care Med 30:2509-2514

39. Thompson JS, Bragg LE, West WW (1990) Serum enzyme levels during intestinal ischemia. Ann Surg 211:369-373

40. Trzeciak S, Dellinger RP, Chansky ME et al (2007) Serum lactate as a predictor of mortality in patients with infection. Intensive Care Med 33:970977

41. Valente S, Lazzeri C, Vecchio S et al (2007) Predictors of in-hospital mortality after percutaneous coronary intervention for cardiogenic shock. Int J Cardiol 114:176-182

42. Van Geloven AA, Biesheuvel TH, Luitse JS et al (2000) Hospital admissions of patients aged over 80 with acute abdominal complaints. Eur J Surg 166:866-871

43. Van Noord D, Mensink PB, De Knegt RJ et al (2011) Serum markers and intestinal mucosal injury in chronic gastrointestinal ischemia. Dig Dis $\mathrm{Sci}$ 56:506-512

44. Vermeulen RP, Hoekstra M, Nijsten MW et al (2010) Clinical correlates of arterial lactate levels in patients with ST-segment elevation myocardial infarction at admission: a descriptive study. Crit Care 14:R164

45. Vernon C, Letourneau JL (2010) Lactic acidosis: recognition, kinetics, and associated prognosis. Crit Care Clin 26:255-283 (table of contents)

46. Vollmar B, Menger MD (2011) Intestinal ischemia/reperfusion: microcirculatory pathology and functional consequences. Langenbecks Arch Surg 396:13-29

47. Wheaton WW, Chandel NS (2011) Hypoxia. 2. Hypoxia regulates cellular metabolism. Am J Physiol Cell Physiol 300:C385-C393

48. Wolf AM, Henne-Bruns D (2003) Mesenteric ischemia. Surgical epidemiology - when to take it into consideration? Chirurg 74:395-398
49. Woods HF, Cohen R (1976) Clinical and biochemical aspects of lactic acidosis. Blackwell Scientific, Oxford

50. Yanar H, Taviloglu K, Ertekin C et al (2007) Planned second-look laparoscopy in the management of acute mesenteric ischemia. World J Gastroenterol 13:3350-33
Chirurg 2015 $\cdot 86: 55$

DOl 10.1007/s00104-014-2906-8

Online publiziert: 24. Januar 2015

(c) Springer-Verlag Berlin Heidelberg 2015

M. Reichert ${ }^{1} \cdot$ M. Hecker ${ }^{2} \cdot$ R. Hörbelt ${ }^{1}$

S. Lerner ${ }^{1} \cdot$ J. Holler ${ }^{1}$. C.M. Hecker ${ }^{3}$

W. Padberg ${ }^{1} \cdot$ M.A. Weigand ${ }^{4}$

A. Hecker ${ }^{1}$

${ }^{1}$ Klinik für Allgemein-, Viszeral-, Thorax-,

Transplantations- und Kinderchirurgie,

Universitätsklinik Gießen und Marburg $\mathrm{GmbH}_{\text {, }}$

Standort Gießen

${ }^{2}$ University of Gießen Lung Center, Medizinische

Klinik 2, Universitätsklinik Gießen und

Marburg $\mathrm{GmbH}$, Standort Gießen

${ }^{3}$ Abteilung für systemische Zellbiologie,

Max Planck Institut für molekulare Physiologie,

Dortmund

${ }^{4}$ Klinik für Anästhesie, Intensivmedizin und

Schmerztherapie, Universitätsklinik Gießen

und Marburg GmbH, Standort Gießen

\section{Erratum zu: Die Rolle von Biomarkern in der Diagnostik der akuten Mesenterialischämie}

In diesem Beitrag wurde der Name der Autorin J. Holler falsch angegeben. Wir bitten zukünftig die korrekte Autorenangabe zu beachten und den Fehler zu entschuldigen.

Die Redaktion

\section{Korrespondenzadresse}

\section{Dr. A. Hecker}

Klinik für Allgemein-, Viszeral-, Thorax-, Transplantations- und Kinderchirurgie, Universitätsklinik Gießen und Marburg GmbH, Standort Gießen,

Rudolf Buchheim Str. 7, 35392 Gießen andreas.hecker@chiru.med.uni-giessen.de
Kommentieren Sie diesen Beitrag auf springermedizin.de

- Geben Sie hierzu den Beitragstitel in die Suche ein und nutzen Sie anschließend die Kommentarfunktion am Beitragsende.
Die Online-Version des Originalartikels können Sie unter http://dx.doi.org/10.1007/ s00104-014-2887-7 finden. 